\title{
Analysis of $\sigma^{54}$-dependent genes in Enterococcus faecalis: a mannose PTS permease (EIIMan) is involved in sensitivity to a bacteriocin, mesentericin Y105
}

\author{
Yann Héchard, Christelle Pelletier, Yves Cenatiempo and Jacques Frère
}

Laboratoire de Microbiologie Fondamentale et Appliquée, CNRS FRE 2224, IBMIG, UFR Sciences, 40 avenue du Recteur Pineau, 86022 Poitiers Cedex, France
Author for correspondence: Yann Héchard. Tel: +33 5494540 07. Fax: +33 549453503. e-mail: yann.hechard@univ-poitiers.fr

The $\sigma^{54}$ RNA polymerase subunit has a prominent role in susceptibility of Listeria monocytogenes and Enterococcus faecalis to mesentericin Y105, a class lla bacteriocin. Consequently, $\sigma^{54}$-dependent genes as well as specific activators also required for expression of these genes were sought. Five putative $\sigma^{54}$ associated activators were detected in the genome of $E$. faecalis V583, and all but one could activate the transcription of permease genes belonging to sugar phosphotransferase systems (PTSs). Interestingly, these activators display a helicase signature not yet reported in this activator family, which could explain the ATP-dependent mechanism of DNA unwinding preceding the start of transcription. To find which activator is linked to susceptibility of $E$. faecalis to mesentericin $Y 105$, their respective genes were subsequently interrupted. Among them, only mptR gene interruption led to a resistance phenotype. Immediately downstream from $m p t R$, a putative $\sigma^{54}$-dependent operon was found to encode a mannose PTS permease, namely Ell Man. Moreover, in liquid culture, glucose and mannose induced the sensitivity of $E$. faecalis to mesentericin Y105. Since sugars have previously been reported to induce PTS permease expression, it appears that Ell $t_{t}^{\text {Man }}$ expression, presumably induced in the presence of glucose and mannose, leads to an enhanced sensitivity of $E$. faecalis to the bacteriocin. Additional information was gained from knockouts within the permease operon. Interruption of the distal mptD gene, which encodes the IID subunit of Ellman, strikingly led to resistance to mesentericin Y105. Moreover, MptD appears to be a peculiar membrane subunit, bearing an additional domain compared to most known IID subunits. According to these results, EIIMan is clearly involved in susceptibility to mesentericin Y105 and could even be its receptor at the $E$. faecalis surface. Finally, it is hypothesized that MptD could be responsible for the targeting specificity, via an interaction between its additional domain and mesentericin Y105.

Keywords: antagonism, subclass IIa, phosphotransferase, sugar, helicase

\section{INTRODUCTION}

Lactic acid bacteria are largely used in food fermentation and have been subjected to numerous studies, leading to the description of many antibacterial peptides, named bacteriocins (Jack et al., 1995; Klaenhammer, 1993). Consequently, the latter or their producing strains are of

Abbreviations: 2DG, 2-deoxyglucose; PTS, phosphotransferase system. great interest in food protection. For example, nisin (Breukink \& de Kruijff, 1999), a bacteriocin produced by Lactococcus lactis, has been used as a food preservative since 1957 . Nisin has a broad spectrum of inhibition against several foodborne Gram-positive pathogens, e.g. Listeria monocytogenes and Clostridium botulinum. It belongs to the class I bacteriocins, termed lantibiotics, which are post-translationally modified peptides. Among other families, subclass IIa bacteriocins deserve special interest as they share an anti-Listeria 
activity and have a conserved structural motif (YGNGVXC) (for a review see Ennahar et al., 2000). They were reported to act by permeabilizing the membrane of susceptible bacteria (Abee, 1995), although their molecular mode of action is largely unknown. For example, the need of a protein receptor at the bacterial surface is still debated. We focused on mesentericin Y105, a subclass IIa bacteriocin, and we looked for its mode of action at the molecular level (Héchard et al., 1992; Maftah et al., 1993). We found that the $\sigma^{54}$ transcription factor is involved in sensitivity of $L$. monocytogenes (Robichon et al., 1997) and Enterococcus faecalis (Dalet et al., 2001) to mesentericin Y105, suggesting that $\sigma^{54}$ directs the expression of an essential protein for sensitivity. $\sigma$ factors are subunits of bacterial RNA polymerase holoenzymes responsible for recognition of promoters. Among the $\sigma$ family, $\sigma^{54}$ is a unique factor (Merrick, 1993). It recognizes particular promoters having a consensus sequence localized at position $-24 /-12$ from the transcription start site, and requires activator proteins (Buck et al., 2000) to initiate transcription. These activators, referred to as $\sigma^{54}$ associated activators, share a conserved central domain, already used to identify new protein members of this family. The first $\sigma^{54}$ factor described in Gram-positive bacteria is encoded by sigL in Bacillus subtilis (Débarbouillé et al., 1991b). It directs, in association with the activator LevR, transcription of the lev operon encoding a permease of the phosphotransferase system (PTS) and the levanase enzyme (Débarbouillé et al., 1991a). Interestingly, two PTS permeases were recently connected with resistance to subclass IIa bacteriocins of L. monocytogenes spontaneous mutants. In the first study, a mannose IIAB PTS component was absent in a leucocin-A-resistant strain (Ramnath et al., 2000), while in the second, a $\beta$-glucoside PTS permease was shown to be overexpressed in a pediocin-PA1-resistant strain (Gravesen $e t$ al., 2000). The PTS transfers a phosphoryl group from phosphoenolpyruvate to sugar via EI, Hpr and EII enzymes (Postma et al., 1993; Saier \& Reizer, 1994). The latter, EII, is a PTS permease involved in transport and concomitant phosphorylation of sugars. EII is composed of three subunits: IIA and IIB, which are cytoplasmic subunits involved in phosphorylation, and IIC, a membrane protein responsible for sugar transport. An additional membrane subunit, IID, is specifically found within the PTS permease of the mannose family.

Interestingly, most of the subclass IIa bacteriocins were described to be also active against another pathogenic species, E. faecalis, in which the $\sigma^{54}$ factor is involved in sensitivity (Dalet et al., 2001). Consequently, $\sigma^{54}$ associated activators and $\sigma^{54}$-dependent genes were sought and analysed. Knockout of these different genes was then achieved to study their involvement in $E$. faecalis sensitivity to mesentericin Y105.

\section{METHODS}

Bacterial strains and growth conditions. E. faecalis V583 or $\mathrm{JH} 2-2$ and its derivatives were grown at $37^{\circ} \mathrm{C}$ in brain-heart infusion (BHI) or in Luria-Bertani (LB) media supplemented or not with sugar. Escherichia coli XL-1 Blue, used for molecular cloning, was grown at $37^{\circ} \mathrm{C}$ in LB medium with vigorous shaking. Erythromycin $\left(5 \mu \mathrm{g} \mathrm{ml}^{-1}\right)$, kanamycin $\left(50 \mu \mathrm{g} \mathrm{ml}^{-1}\right)$ or ampicillin $\left(100 \mu \mathrm{g} \mathrm{ml}^{-1}\right)$ was added, as needed. Leuconostoc mesenteroides Y105, which produced mesentericin Y105, was grown in Man-Rogosa-Sharp (MRS) medium at $30^{\circ} \mathrm{C}$.

DNA manipulations and gene interruption. Molecular cloning and DNA manipulations were performed as described previously (Sambrook et al., 1989). Restriction and modification enzymes purchased from 'Life technologies' were used as recommended by the manufacturer. Internal gene fragments, used for knockout experiments, were amplified by PCR with the following primers bearing a HindIII site: $m p o R$ primers, GTCAAGCTTCTGGCTATACCCG and TTTTAAGCTTCGCCCATCCGTG; $m p t R$ primers, GTAAAGCTTTGAATCAACTGGTTCG and CAGAAAGCTTCCATCTGCTTCATC; $m p h R$ primers, TCAAAGCTTCCAAGAAATGATCGATG and TGGCAAAGCTTAACCGACACG; lpoR primers, AGTAAGCTTCGGACAAGTCAGCG and TCCAAGCTTAATGGCAAAAGCAGATG; $m p t B$ primers, CGTTAAGCTTGAATTGATGATCG and AATAAGCTTGGCTGTCTGCTGC; $m p t D$ primers, AGCTGAAGCTTGGCGTTCAAC and AGAACAAGCTTTGTAACCAAACTC. The resulting PCR fragments were digested with HindIII and ligated at the same site in pUCB300 (Frère et al., 1993), a non-replicative plasmid bearing an erythromycin-resistance gene. It gave rise to the pEF17 (' $m p o R$ '), pEF16 (' $m p t R$ '), pEF23 ('mphR'), pEF5 ('lpoR'), pEF18 ('mptB') and pEF19 ('mptD') plasmids. They were then used to transform $E$. faecalis JH2-2 as described by Wyckoff et al. (1991) to achieve independent gene knockout by homologous recombination with the E. faecalis JH2-2 chromosome. The resulting interrupted mutants (erythromycin-resistant) from each experiment were analysed by Southern blotting of chromosomal DNA (Sambrook et al., 1989), previously digested by HindIII. The DNA probes used for hybridization were synthesized by random priming from the PCR fragments described above.

2-Deoxyglucose-resistant strain selection. E. faecalis JH2-2 was grown on LB agar plates supplemented with fructose at $2 \mathrm{~g} \mathrm{l}^{-1}$, as a carbon source, and 2-deoxyglucose (2DG), a nonmetabolizable analogue of glucose, at $10 \mathrm{mM}$. The resulting colonies, corresponding to the growth of 2DG-resistant mutants, were isolated twice on the same medium. 2-DG is a toxic molecule that enters bacteria via a PTS permease of the mannose family (Bond et al., 1999). Consequently, this permease is not usually expressed in 2DG-resistant mutants.

Bacteriocin purification and assays. Mesentericin Y105, produced by Leuconostoc mesenteroides Y105, was purified as previously described (Guyonnet et al., 2000). E. faecalis susceptibility was assayed by spot on lawn or microtitre plate tests. The former was achieved by overlaying a BHI agar plate $(1.5 \%)$ with a BHI agar lawn $(0.7 \%)$ inoculated at $1 \%$ with an E. faecalis culture. Purified mesentericin Y105 $(5 \mu \mathrm{l})$ was spotted on the lawn and the plate was then incubated overnight at $37^{\circ} \mathrm{C}$ before inhibition zones were noted. The microtitre plate assay was carried out by inoculating $200 \mu \mathrm{l}$ BHI or LB supplemented with various sugars at $2 \mathrm{~g} \mathrm{l}^{-1}$. Bacterial growth was monitored by measurement of the $\mathrm{OD}_{620}$ and $5 \mu \mathrm{l}$ purified mesentericin Y105 was added when the $\mathrm{OD}_{620}$ reached $0 \cdot 1$.

DNA sequencing. Cycle sequencing was achieved with the ABI Prism BigDye Terminator Cycle Sequencing Ready Reaction kit (Perkin-Elmer) and analysed with the ABI Prism 310 genetic analyser. Sequence data from E. faecalis V583 were 
obtained from the Institute for Genomic Research through the website at http://www.tigr.org/.

\section{RESULTS}

\section{Computer analysis of the $\sigma^{54}$-dependent genes}

Neither $\sigma^{54}$-associated activators nor $\sigma^{54}$-dependent genes have been reported so far in E. faecalis. We first looked for $\sigma^{54}$-associated activator genes within the $E$. faecalis V583 genome, taking advantage of two main features: one, the activator genes are often found upstream from the $\sigma^{54}$-dependent genes they control; and two, the activator proteins have a highly conserved central domain, used for their identification (Studholme \& Buck, 2000). Interestingly, shotgun sequencing of $E$. faecalis V583 has been completed and closure of gaps was under way, providing sufficient information for our study.

Thus, we first used an amino acid sequence of the central domain to screen the E. faecalis V583 genome. BLAST results displayed five high scores ( $>45 \%$ identities), corresponding to five ORFs, named $m p o R, m p t R$, $m p h R, l p o R$ and $x p o R$. These genes potentially encode $\sigma^{54}$-associated activators of 937, 961, 923, 901 and 403 amino acids, respectively. The latter, $x p o R$, is interrupted by a sequence which is identical to the transposon Tn4001 from Staphylococcus aureus (Byrne et al., 1989). Interestingly, all these activators share highest identities, about $30 \%$, with an unusual member of the $\sigma^{54}$ associated activator family, LevR of $B$. subtilis (Débarbouillé et al., 1991a). Besides the classical motifs usually described in $\sigma^{54}$-associated activators, the central domain of all these activators displayed a DEAH motif, according to Prosite (Fig. 1), usually found in helicases. Such similarities with helicases have already been looked for in activators to explain their involvement in DNA conformational changes (Buck et al., 2000), but none have been found. We suggest that, owing to the presence of the DEAH motifs, these activators could be directly responsible for ATP-dependent DNA unwinding, allowing initiation of transcription. In addition, we found degenerate DEAH motifs in all the other $\sigma^{54}$ associated activators so far described in the database (data not shown), indicating that they could also bear a helicase activity.

We then looked, downstream of the activator genes, for the $-24 /-12$ consensus sequence (TTGGCACNNNNNTTGCT), identifying a promoter recognized by $\sigma^{54}$. A $\sigma^{54}$ promoter was found just downstream of every activator gene, except the truncated $x p o R$, therefore preceding a putative $\sigma^{54}$-dependent operon. Four ORFs were found in each operon downstream of $m p o R, m p t R$ and $m p h R$ (Fig. 2). These ORFs putatively encode proteins that display highest similarities with PTS permeases (EII) of the mannose family, i.e. IIA ${ }^{\text {Man }}$, IIB $^{\text {Man }}$, IIC ${ }^{\text {Man }}$ or IID ${ }^{\text {Man }}$ subunits (a fused IIAB ${ }^{\text {Man }}$ subunit is encoded by $m p t A$ in the $m p t$ operon). Finally, downstream of $l_{p o} R$, two $\sigma^{54}$ promoter sequences were found, respectively followed by three ORFs that putatively encode proteins with highest similarities with PTS permease of the lactose family, i.e. IIA ${ }^{\mathrm{Lac}}$ IIB $^{\mathrm{Lac}}$ and IIC ${ }^{\text {Lac }}$ subunits. Surprisingly, all these $\sigma^{54}$-dependent operons probably encode a PTS permease, while to date only one $\sigma^{54}$-dependent PTS permease has been described (Débarbouillé et al., 1991a). This is a fructose permease encoded by the lev operon and controlled by the LevR activator in B. subtilis.

\section{Role of $\sigma^{54}$-associated activators in sensitivity to mesentericin Y105}

E. faecalis V583 and JH2-2 were both sensitive to mesentericin Y105. However, further experiments were performed only with JH2-2, since our previous studies on $\sigma^{54}$ involvement were conducted with the latter strain. Each activator gene, i.e. $m p o R, m p t R, m p h R$ and lpoR, was knocked-out in E. faecalis JH2-2 to assess its involvement in mesentericin Y105 sensitivity. To this aim, an internal fragment of each gene was amplified by PCR from JH2-2 genomic DNA and cloned in pUCB300. The resulting plasmids were independently used to transform E. faecalis $\mathrm{JH} 2-2$ and led to homologous

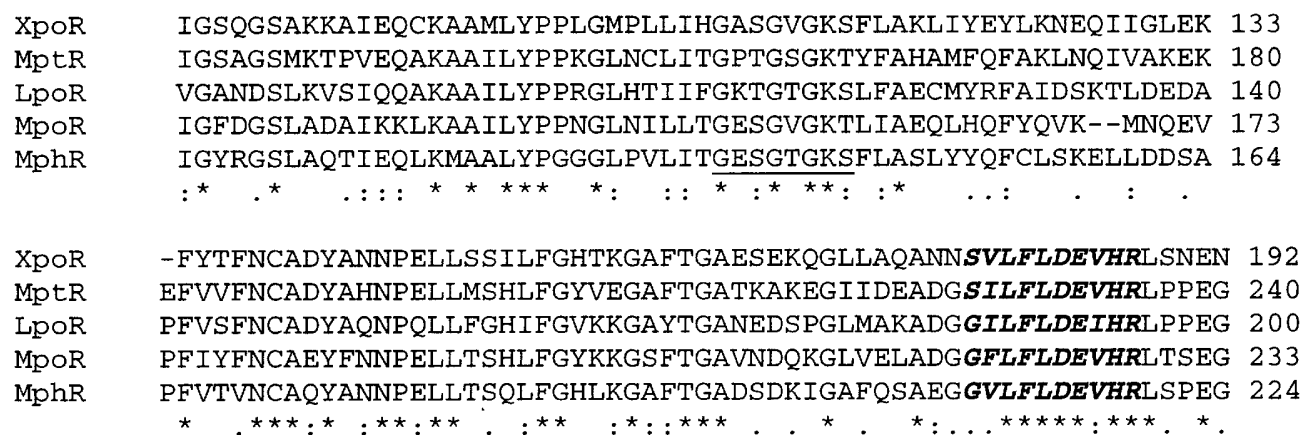

Fig. 1. Alignment of part of the conserved central domain found within putative $\sigma^{54}$-associated activators of $E$. faecalis V583 (ClustalW program). The putative ATP/GTP-binding sites (Prosite PDOC00017) are underlined and the putative DEAH-box subfamily ATP-dependent helicase signatures (Prosite PDOC00039) are in bold and in italics. Identical residues in all sequences in the alignment are indicated by an asterisk (*); conserved substitutions are indicated by a colon (:) and semi-conserved substitutions are indicated by a stop (.). 


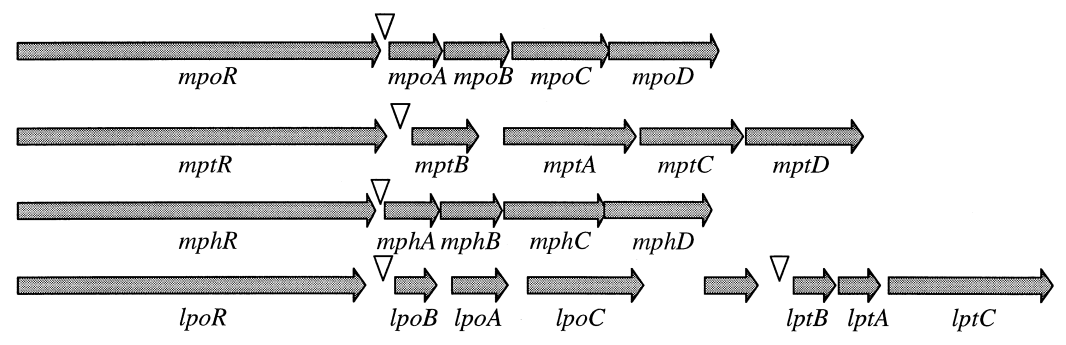

Fig. 2. Organization of $\sigma^{54}$-associated activator genes and $\sigma^{54}$-dependent PTS operons. The inverted triangles represent the putative 2000 4000 6000 8000 $-24 /-12$ promoters.

Table 1. Sensitivity of $E$. faecalis strains to purified mesentericin $Y 105$, assessed by a spot on lawn assay

\begin{tabular}{|lc|}
\hline Strain & $\begin{array}{r}\text { Diameter of inhibition } \\
\text { zone (mm) }\end{array}$ \\
\hline E. faecalis V583 (WT) & 11 \\
E. faecalis JH2-2 (WT) & 12 \\
JH1 (rpoN) & 0 \\
JH3 (lpoR) & 12 \\
JH6 (2DG-resistant) & 0 \\
JH7 (mptR) & 0 \\
JH8 (mpoR) & 12 \\
JH9 (mptB) & 0 \\
JH10 (mptD) & 0 \\
JH11 (mphR) & 12 \\
\hline
\end{tabular}

recombination in the chromosome. Knockout of $m p o R$, $m p t R, m p h R$ and $l p o R$, verified by both PCR and Southern blotting (data not shown), led to the JH8, JH7, JH11 and JH3 strains, respectively.

The sensitivity of these knockout strains was then tested by a spot on lawn assay with purified mesentericin Y105 (Table 1). E. faecalis JH3, JH8 and JH11 remained sensitive to mesentericin Y105, similarly to the wild-type $\mathrm{JH} 2-2$ strain. In contrast, the JH7 strain (knockout of $m p t R$ ) became fully resistant to purified mesentericin Y105, as was the previously described JH1 strain $\left(\sigma^{54}\right.$ deficient mutant) (Dalet et al., 2001). Consequently, MptR is the only E. faecalis $\sigma^{54}$-associated activator presumably involved in sensitivity to mesentericin Y105. This suggests that MptR and $\sigma^{54}$ control the expression of proteins involved in E. faecalis sensitivity to mesentericin Y105.

\section{Role of Ell $t_{t}^{\text {Man }}$ in sensitivity to mesentericin Y105}

$\sigma^{54}$-associated activator genes were often found just upstream of the operon controlled by the activator. Downstream of $m p t R$, four ORFs flanked by two hairpin loops with a calculated free energy of -84 and $-109 \cdot 2 \mathrm{~kJ}$, respectively, probably compose an operon, named $m p t$. As described above, these ORFs putatively encode four proteins that display highest similarities with PTS permease components of the mannose family.
They were consequently named $\mathrm{MptB}$ (170 aa), MptA (342 aa), MptC (267 aa) and MptD (303 aa). Knockout of $m p t B$ and $m p t D$, the proximal and the distal genes of the $m p t$ operon, was achieved independently by homologous recombination, leading to the $\mathrm{JH} 9$ and $\mathrm{JH} 10$ mutants, respectively. These knockout mutants were tested for sensitivity to mesentericin Y105. They were both resistant to mesentericin Y105, as were MptR- and $\sigma^{54}$-deficient mutants (Table 1 ), which supports the operon $m p t$ being involved in sensitivity and being controlled by MptR and $\sigma^{54}$. Moreover, since knockout of $m p t D$, the distal gene of the operon, led to resistance, the corresponding MptD protein should play a crucial role in sensitivity. In this respect, the resistance of $\mathrm{JH} 9$ (knockout of $m p t B$ ) was probably due to the lack of $m p t D$ expression through a polar effect. Interestingly, MptD displays an additional domain compared to the other IID subunits found within the E. faecalis genome (Fig. 3) and to the other IID subunits reported in the GenBank database, except ManN of Streptococcus salivarius. Moreover, according to structure prediction with the TMpred program, this additional domain could be located within an extracellular loop. We thus hypothesize that this motif could be involved in mesentericin Y105 targeting of the MptD subunit, partly explaining the narrow spectrum of mesentericin Y105 activity.

Finally, E. faecalis 2DG-resistant strains were isolated on selective medium. Among them, six clones (JH6-1 to JH6-6) were tested for sensitivity to mesentericin Y105 by a spot on lawn assay. All of them were resistant to mesentericin Y105 (Table 1). This result emphasizes that an $\mathrm{EII}^{\mathrm{Man}}$ of E. faecalis, possibly $\mathrm{EII}_{\mathrm{t}}^{\mathrm{Man}}$, is actually involved in sensitivity to mesentericin Y105, because 2DG-resistant strains are known to be defective in EII $^{\text {Man }}$ expression (Veyrat et al., 1994).

\section{Sugar effects on E. faecalis JH2-2 sensitivity to mesentericin Y105}

Since E. faecalis JH2-2 sensitivity to mesentericin Y105 seems to be linked to a specific PTS mannose permease, the effect of various sugars on sensitivity was tested. Indeed, expression of PTS permeases is specifically induced by the transported sugar (Saier \& Reizer, 1994). E. faecalis JH2-2 was grown in LB medium supplemented independently with the following sugars at 
Fig. 3. Alignment of putative extracellular loops of IID subunits of $\sigma^{54}$-dependent mannose PTS permeases. The additional domain of MptD is in bold.

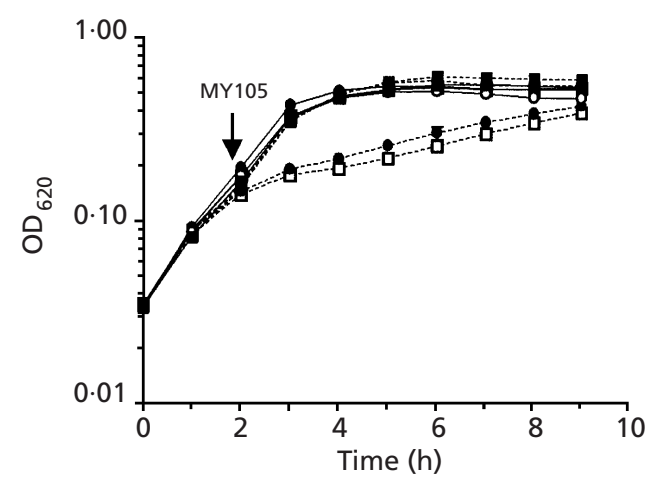

Fig. 4. Effect of several sugars on $E$. faecalis $\mathrm{JH} 2-2$ sensitivity to mesentericin $\mathrm{Y} 105$. E. faecalis was grown in LB supplemented with cellobiose $(\square)$, fructose $(\bigcirc)$, glucose $(\mathbf{O})$ or mannose $(\square)$ at $2 \mathrm{~g} \mathrm{I}^{-1}$. The dotted lines represent growth curves in the presence of mesentericin Y105 (addition is indicated by an arrow) and the unbroken lines represent growth curves in the absence of mesentericin Y105.

$2 \mathrm{~g} \mathrm{l}^{-1}$ : fructose, cellobiose, glucose and mannose. Fig. 4 shows that the sensitivity of E. faecalis was highly increased in the presence of glucose or mannose, compared to cellobiose or fructose. E. faecalis was also weakly sensitive to mesentericin Y105 in LB medium without any added sugar (data not shown). This suggests that glucose and mannose activate the expression of a protein involved in sensitivity to mesentericin Y105, probably the $\mathrm{EII}_{\mathrm{t}}^{\mathrm{Man}}$ PTS permease.

\section{DISCUSSION}

We had previously shown that the $\sigma^{54}$ factor of $E$. faecalis is involved in sensitivity to mesentericin Y105 and various subclass IIa bacteriocins (Dalet et al., 2001). Consequently, we studied the $\sigma^{54}$ regulon, via the characterization of five $\sigma^{54}$-associated activators and several $\sigma^{54}$-dependent genes. Although previous sequence analysis of $\sigma^{54}$-associated activators did not reveal any similarity with helicases (Buck et al., 2000), we found that in E. faecalis all of them bear a helicase motif (DEAH box). This helicase motif, together with the nucleotide binding site, could be involved in ATPdependent DNA unwinding that promotes initiation of transcription.

According to the acquired resistance phenotype displayed by knockout mutants, MptR appears as the only one, among the five activators, to be involved in sensitivity to mesentericin Y105. MptR probably activates the transcription, together with $\sigma^{54}$, of the flanking downstream operon, which encodes a mannose PTS permease, $\mathrm{EII}_{\mathrm{t}}^{\text {Man }}$. In addition, knockout within this operon also led to resistance, which supports $\mathrm{EII}_{\mathrm{t}}^{\mathrm{Man}}$ being involved in resistance and being dependent on MptR. We have also recently shown that knockout within an operon encoding a $\sigma^{54}$-dependent PTS permease of the mannose family leads to L. monocytogenes resistance (K. Dalet, Y. Cenatiempo, P. Cossart, The European Listeria Genome Consortium and Y. Héchard, unpublished results). Moreover, the IIAB subunit of a mannose PTS permease was shown to be absent in a spontaneous mutant of $L$. monocytogenes resistant to leucocin A (Ramnath et al., 2000), favouring the lack of EII ${ }^{\mathrm{Man}}$ expression. Another spontaneous mutant of L. monocytogenes, resistant to pediocin PA-1 (a subclass IIa bacteriocin), has been reported by others to overexpress a PTS permease from the $\beta$-glucoside family (Gravesen et al., 2000). However, the same group found out that knockout of the $\beta$-glucoside operon did not modify the sensitivity to pediocin PA-1 and that this PTS is also overexpressed in our L. monocytogenes rpoN mutant, lacking $\sigma^{54}$ (A. L. Gravesen, personal communication). These results on both E. faecalis and L. monocytogenes point towards an essential role of an EII $^{\mathrm{Man}}$ permease in sensitivity to mesentericin Y105 and related subclass IIa bacteriocins. Our current hypothesis is that $\mathrm{EII}^{\mathrm{Man}}$ is probably a receptor for these bacteriocins. Finally, we showed that the presence of glucose and mannose in the culture medium greatly increases E. faecalis sensitivity to mesentericin Y105. Since PTS permease expression has been reported to be specifically induced by transported sugars, we hypothesize that glucose and mannose induce the expression of $\mathrm{EII}_{\mathrm{t}}^{\mathrm{Man}}$, thereby leading to an increase in the number of potential protein receptors for mesentericin Y105.

Further data favour the above hypotheses and focus on a particular component of the permease. Knockout of $m p t D$, the distal gene of this operon, led to resistance and thus the corresponding MptD subunit seems essential in E. faecalis sensitivity to mesentericin Y105. In L. monocytogenes, knockout or in-frame deletion of a gene, encoding a IID ${ }^{\mathrm{Man}}$ subunit, also led to resistance (K. Dalet, Y. Cenatiempo, P. Cossart, The European Listeria Genome Consortium and Y. Héchard, unpublished results). Moreover, this IID ${ }^{\text {Man }}$ subunit of $L$. monocytogenes harbours an additional motif, as found here in E. faecalis $\mathrm{MptD}$, and the above-mentioned inframe deletion removed this domain. Thus, in these two organisms, the IID subunit of the EII ${ }^{\mathrm{Man}}$ involved in sensitivity bears an additional motif (a putative external loop) not found within the other IID subunits described, except ManN of $S$. salivarius. The additional domain of E. faecalis shares $64 \%$ similarity with that of $L$. monocytogenes and only $39 \%$ with that of S. salivarius. Moreover, IID subunits are integral membrane proteins and could therefore interact directly with mesentericin 
Y105. Accordingly, we propose that an EII ${ }^{\mathrm{Man}}$ is a receptor for various subclass IIa bacteriocins in $L$. monocytogenes and E. faecalis and that its IID Man component plays a central role in bacteriocin action, probably by a direct protein-protein interaction involving the additional domain.

Whether an EII ${ }^{\text {Man }}$ is also implicated in sensitivity to subclass IIa bacteriocins when present in other bacteria is being analysed. Direct protein-protein interaction between subclass IIa bacteriocins and the permease will be further investigated, together with the implication of the additional domain found in their IID components.

\section{ACKNOWLEDGEMENTS}

Sequencing of E. faecalis V583 was accomplished with support from the National Institute of Allergy and Infectious Diseases. We thank Dr Gilmore, Oklahoma City, for providing us with E. faecalis V583. This work was partly supported by a partnership with the Rhodia Food company.

\section{REFERENCES}

Abee, T. (1995). Pore-forming bacteriocins of gram-positive bacteria and self-protection mechanisms of producer organisms. FEMS Microbiol Lett 129, 1-10.

Bond, D. R., Tsai, B. M. \& Russell, J. B. (1999). Physiological characterization of Streptococcus bovis mutants that can resist 2deoxyglucose-induced lysis. Microbiology 145, 2977-2985.

Breukink, E. \& de Kruijff, B. (1999). The lantibiotic nisin, a special case or not? Biochim Biophys Acta 1462, 223-234.

Buck, M., Gallegos, M. T., Studholme, D. J., Guo, Y. \& Gralla, J. D. (2000). The bacterial enhancer-dependent sigma $(54)(\operatorname{sigma}(\mathrm{N}))$ transcription factor. J Bacteriol 182, 4129-4136.

Byrne, M. E., Rouch, D. A. \& Skurray, R. A. (1989). Nucleotide sequence analysis of IS256 from the Staphylococcus aureus gentamicin-tobramycin-kanamycin-resistance transposon Tn4001. Gene 81, 361-367.

Dalet, K., Briand, C., Cenatiempo, C. \& Héchard, Y. (2001). The rpoN gene of Enterococcus faecalis directs sensitivity to subclass IIa bacteriocins. Curr Microbiol 41, 441-443.

Débarbouillé, M., Martin-Verstraete, I., Klier, A. \& Rapoport, G. (1991a). The transcriptional regulator LevR of Bacillus subtilis has domains homologous to both sigma 54- and phosphotransferase system-dependent regulators. Proc Natl Acad Sci U S A 88, 2212-2216.

Débarbouillé, M., Martin-Verstraete, I., Kunst, F. \& Rapoport, G. (1991b). The Bacillus subtilis sigL gene encodes an equivalent of sigma 54 from gram-negative bacteria. Proc Natl Acad Sci U S A 88, 9092-9096.

Ennahar, S., Sashihara, T., Sonomoto, K. \& Ishizaki, A. (2000). Class IIa bacteriocins: biosynthesis, structure and activity. FEMS Microbiol Rev 24, 85-106.

Frère, J., Benachour, A., Novel, M. \& Novel, G. (1993). Identification of the theta-type minimal replicon of the Lactococcus lactis subsp. lactis CNRZ270 lactose protease plasmid pUCL22. Curr Microbiol 27, 97-102.
Gravesen, A., Warthoe, P., Knochel, S. \& Thirstrup, K. (2000). Restriction fragment differential display of pediocin-resistant Listeria monocytogenes 412 mutants shows consistent overexpression of a putative $\beta$-glucoside-specific PTS system. Microbiology 146, 1381-1389.

Guyonnet, D., Fremaux, C., Cenatiempo, Y. \& Berjeaud, J.-M. (2000). Method for rapid purification of class IIa bacteriocins and comparison of their activities. Appl Environ Microbiol 66, $1744-1748$.

Héchard, Y., Dérijard, B., Letellier, F. \& Cenatiempo, Y. (1992). Characterization and purification of mesentericin Y105, an antiListeria bacteriocin from Leuconostoc mesenteroides. J Gen Microbiol 138, 2725-2731.

Jack, R. W., Tagg, J. R. \& Ray, B. (1995). Bacteriocins of grampositive bacteria. Microbiol Rev 59, 171-200.

Klaenhammer, T. R. (1993). Genetics of bacteriocins produced by lactic acid bacteria. FEMS Microbiol Rev 12, 39-85.

Maftah, A., Renault, D., Vignoles, C., Héchard, Y., Bressollier, P., Ratinaud, M. H., Cenatiempo, Y. \& Julien, R. (1993). Membrane permeabilization of Listeria monocytogenes and mitochondria by the bacteriocin mesentericin Y105. J Bacteriol 175, 3232-3235.

Merrick, M. J. (1993). In a class of its own - the RNA polymerase sigma factor sigma 54 (sigma N). Mol Microbiol 10, 903-909.

Postma, P. W., Lengeler, J. W. \& Jacobson, G. R. (1993). Phosphoenolpyruvate : carbohydrate phosphotransferase systems of bacteria. Microbiol Rev 57, 543-594.

Ramnath, M., Beukes, M., Tamura, K. \& Hastings, J. W. (2000). Absence of a putative mannose-specific phosphotransferase system enzyme IIAB component in a leucocin A-resistant strain of Listeria monocytogenes, as shown by two-dimensional sodium dodecyl sulfate-polyacrylamide gel electrophoresis. Appl Environ Microbiol 66, 3098-3101.

Robichon, D., Gouin, E., Débarbouillé, M., Cossart, P., Cenatiempo, Y. \& Héchard, Y. (1997). The rpoN (sigma54) gene from Listeria monocytogenes is involved in resistance to mesentericin Y105, an antibacterial peptide from Leuconostoc mesenteroides. J Bacteriol 179, 7591-7594.

Saier, M. H., Jr \& Reizer, J. (1994). The bacterial phosphotransferase system: new frontiers 30 years later. Mol Microbiol $13,755-764$.

Sambrook, J., Fritsch, E. F. \& Maniatis, T. (1989). Molecular Cloning: a Laboratory Manual, 2nd edn. Cold Spring Harbor, NY : Cold Spring Harbor Laboratory.

Studholme, D. J. \& Buck, M. (2000). The biology of enhancerdependent transcriptional regulation in bacteria: insights from genome sequences. FEMS Microbiol Lett 186, 1-9.

Veyrat, A., Monedero, V. \& Perez-Martinez, G. (1994). Glucose transport by the phosphoenolpyruvate:mannose phosphotransferase system in Lactobacillus casei ATCC 393 and its role in carbon catabolite repression. Microbiology 140, 1141-1149.

Wyckoff, H. A., Sandine, W. E. \& Kondo, J. K. (1991). Transformation of dairy Leuconostoc using plasmid vectors from Bacillus, Escherichia and Lactococcus hosts. J Dairy Sci 74, 1454-1460.

Received 1 November 2000; revised 6 January 2001; accepted 11 January 2001. 